in seinen text! Eine drastischere illustration für die mechanische art der interpolation der ags. reihe in die nordische genealogie hätte sich wol kaum erwarten lassen. Man darf hiernach sicher behaupten, dass von einer auch nur einigermassen volkstimlichen tradition nicht die rede sein kann, welche etwa die ags. namen in die nordischen quellen gebracht hätte; vielmehr handelt es sich in erster instanz um eine rein gelehrte manipulation eines einzelnen, dem dann später andere nachschrieben.

Im einem punkte muss freilich die vorlage noch correcter gewesen sein als unser $\mathrm{H}$, bei dem namen Scealdwa, dessen $d$ in $\mathrm{H}$ fehlt, wenn der abdruck richtig ist. Aber gerade auch bei diesem namen zeigt sich wider wie mechanisch man bei der herübernahme verfuhr. Das Scealdwa der ags. quelle wird der nordische compilator zunächst wol durch Scealdua widergegeben haben (vgl. Beðuig, Beduigg etc. = ags. Bedwiz), dann aber ist das $u$ als $n$ verlesen und so entstand Scealdna im Langfeðgatal, das seinerseits wol wider den ausgangspunkt für Skialldun und Skialldin in SE. und Fl. 1 abgegeben hat.

HALLE a./S., 8. december 1890.

E. SIEVERS.

\title{
SINTARFIZILO.
}

Gegenuber Mullenhoffs deutung des namens Sintarfizilo Zs. fda. 23, $163 \mathrm{f}$. ist $\mathrm{Kögel}$ in Pauls Grundriss $2^{\mathrm{a}}, 185$ wie ich glaube mit recht zu der ältel'en auffassung J. Grimms zurtuckgekehrt, welcher (Zs. fda. 1,4) in -fizilo ein adj. fizil sah. Ein solches adj. ist als simplex bisher nur einmal in den Pariser Vergilglossen Zs. fda. 15, $42=$ Ahd. gl. 2, 709, 5 belegt, und Mullenhoff meinte daber, dass das fix zelaz dieser stelle leicht fur fizziluehaz verschrieben sein könnte:. Es scheint aber noch ein altes und unanfechtbares zeugnis fur das wort an einer bisher ubersehenen stelle, in des Paulus Diaconus Langobardengeschichte 1,24 vorzuliegen.

Als Alboin um sich die waffen zu lholen zu dem Gepidenkönig Turisind gekommen war, dessem sohn Turismod er im kampfe getötet hatte, und beim mahlee auf Turismods platze 
zur rechten Turisinds sass, brach dieser im andenken an den verlust des solins in die schmerzerfullten worte aus: 'Amabilis mihi locus iste est, sed persona quae in eo residet satis ad videndum gravis.' Tunc regis alter qui aderat filius, patris sermone stimulatus, Langobardos iniuriis lacessere coepit, asserens eos qui a suris inferius candidis utebantur fasceolis equabus quibus crure tenus pedes albi sunt similes esse, dicens ' $\mathrm{Fe}$ tilae sunt equae quas similatis'. Tunc unus e Langobardis ad haec ita respondit: 'Perge, ait, in campum Asfeld, ibique procul dubio poteris experiri, quam validae istae quas equas nominas praevalent calcitrare, ubi sic tui dispersa sunt ossa germani quemadmodum vilis iumenti in mediis pratis.' Nur dem energischen einschreiten Turisinds gelingt es den ob dieser schmähungen drohenden kampf zu verhindern.

Dass Waitz im unrecht ist, wenn er, in den anmerkungen an der citierten stelle, fetilae als foetulae deutet, bedarf wol keines beweises; höchstens könnte man versucht sein, in fetilae ein verderbnis für rein lateinisches petilae zu vermuten. Aber auch diese vermutung wtirde, meine ich, haltlos sein, da die gesammte reichliche varietas lectionis: fetiles, fetite, fetule, fecile, fecule, fetide, fert ille, fertiles, facile, faciles, felices den charakteristischen anlaut $f$ für das original feststellt. Merkwurdig aber ist das unverschobene $t$ von fetilus oder fetilis (denn auch die lesart fetiles ist verhältnismässig gut bezeugt; dagegen ist mit dem $c$ von fecile u.s. w. nach der art der handschriftlichen uberlieferung nichts anzufangen: es ist lesefehler für $t$ ). Die von Paulus erzăhlte geschichte soll sich. zwischen 540 und 550 zugetragen haben, also in einer zeit wo auch das $t$ bei den Langobarden wol noch unverschoben war. Aber sollte Paulus in der lage gewesen sein, einen so alten geschriebenen bericht zu benutzen, dass auch in ihm unverschobenes $t$ noch zeitgerecht gewesen wäre? Doch schwerlich. Eher darf man vielleicht an eine mischform im spätlatein Oberitaliens denken. Wie dem aber auch sein mag, auf alle fälle liegt hier ein dem lat. adjectivum petilus gleichbedeutendes germanisches adjectiv zu grunde, das dem ahd. simplex fizzil an die seite $\mathrm{zu}$ stellen ist und dessen existenz sichern hilft, soweit ein neuer beweis neben dem an sich schon genügenden compositum fitịl-vôt (Kögel a. a. o.) noch nötig ist. 
Beachtenswert ist ubrigens bei unserer stelle noch, dass der vergleich der Langobarden mit den fetilae equae als iniuriae bezeichnet wird. Nach der antwort des Langobarden sieht es freilich fast so aus, als ob nur das femininum equae als das schimpfliche empfunden worden sei, da er, das adj. fetilae ignorierend, nur das subst. equae aufnimmt und demgemäss weiter von einem vile iumentum spricht (iumentum bezeichnet im latein dieser zeit auch gewöhnlich eine stute, s. C. Rittweger, De equi vocabulo et cognominatis, Halle 1890, $46 \mathrm{ff}$.). Auf der andern seite muss man aber doch auch wider betonen, dass bei dieser auffassung die eigentliche pointe wegfiele, der spöttische hinweis auf die besondere tracht der Langobarden. War etwa die fetila equa eine besonders gering geschätzte species (vile iumentum)?1) Man könnte sich wol denken, dass die mehrfarbigkeit als kennzeichen verderbter rasse betrachtet worden wäre, und damit wäre dann für den namen Fitela, Sin-fjotli eine neue bedeutsame beziehung geschaffen.

Was aber bedeutet Sintar-fizzilo? Kögel meint 'schlackenscheckig, weiss und schwarz gefleckt wie schlacke oder hammerschlag'. Das halte ich für unmöglich. Bunte schlacken gibt es $\mathrm{ja}$ in menge, aber 'schlacke' an sich ist nicht weiss und schwarz gefleckt, noch weniger der hammerschlag: der ist erst recht einfarbig, dunkel grauschwarz. Kögel hat bei seiner erklärung ausserdem einen umstand ubersehen, auf den bereits die angaben von J. Grimm a. a. o. 6 fuhren mussten, nämlich dass germ. sindra- zunächst nur die glubende, flussige schlacke oder den gluhenden, funkensprtuhendon hammerschlag ${ }^{2}$ ) bezeichnete.

1) Leider kann ich für diese vermutung, germanische belege nicht beibringen. Eine art von analogie ist es aber doch immerhin, dass bei den Römern die varii, zn denen Isidor Orig. 12,1,52 auch die petili stellt, als geringeren ranges betrachtet werden; s. E. Böhmer, Roman. stud. $1,240 \mathrm{ff}$.

2) Streng genommen hat sindra-wol nur die ausgeschmolzene schlacke bezeichnet, nicht den in fester form absplitternden hammerschlag; oder wenn die übertragung auf den 'hammerschlag' im eigentlichen sinne des wortes alt ist, so ist das wol mur folge einer vermengung der beim ersten ausschmieden schlackenhaltiger luppen noch abfliessenden und abspritzenden schlacke mit dem später folgenden hammerschlag. 
Das tritt bei der anwendung des worts in allen einzelsprachen deutlich hervor, und vielfach selbst bis auf die neueste zeit herab. So heisst es SE. 2, 42, dass der giftschlamm der Élivágar allmählich erstarrte sem sindr bat er renn ór eldinum; daher denn auch die kenning sags sindr 'scintilla maris' fur 'gold' (Egilsson 707) und das bereits von J. Grimm betonte sindra 'funken sprtihen, funkeln' (Egilsson 707. Vigfússon 529, auch noch neunorw., Aasen 651), desgl. neuisl. sindri (glänzender) 'kiesel' (Grimm und Egilsson a. a. o.). Im engl. cinder ist die beziehung auf die 'glut' noch heutzutage nicht erloschen (Murray 2, 417 f.), desgl. in dän. sinder. Für das mhd. vgl. dô gloste ich als daz îsen, sô man dâvon siht rîsen in der esse $d a z$ sinder Servat. 3511, heizer danne ein sinder MS. 1, 184 ${ }^{\mathrm{b}}$, ich nâch dir brinne sam in der gluot ein sinder Frl. 416, 7 u. ä. (Mhd. wb. 3, 298 f.). Auch nhd. tritt die bedeutung des gluhens noch oft bei sinter hervor, s. z. b. Frisch 2, 280. Adelung 4, 487. Jacobsson, Technol. wb. 1, 11 unter 'ablassen'. Endlich weist auch dis nhd. sinter 'tropfstein' nebst dem verbum sintern sowol in der bedeutung 'abtropfen, durchsickern' (vgl. dazu wider das technische seigern 'ausschmelzen') wie in der bedeutung 'gerinnen' auf flussigen sintar hin.

Wenn also sintar in Sintarfizilo sich auf die farbe bezieht, so ist damit gewiss nicht die dunkle farbe der erstarrten eisenschlacke gemeint, sondern höchstens ein strahlendes gelb, wie es der geschmolzenen schlacke eigen ist. Damit weiss ich freilich wider nichts anzufangen. Vielleicht gelingt es aber einem andern, aus dem hier gegebenen eine befriedigende deutung $\% u$ finden, und deshalb babe ich auch diese schlussbemerkungen nicht unterdricken wollen.

HALLE a. S., 20. december 1890.

E. SIEVERS.

\section{DIE ANGEBLICHE GÖTTIN RICEN.}

In der Myth. 14, 242 anm. schreibt J. Grimm: 'ich könnte ein ags. Ricen in den text aufnehmen, wenn ich etwas anders von ihr wuste als was Lyes glossar aus Cod. cot. 65, 87 anfubrt: Ricenne Diana. Die bildung ist wie binen (ancilla), 